\title{
Publisher Correction: Precisely printable and biocompatible silk fibroin bioink for digital light processing 3D printing
}

Soon Hee Kim¹, Yeung Kyu Yeon¹, Jung Min Lee, Janet Ren Chao², Young Jin Lee ${ }^{1}$, Ye Been Seo', Md. Tipu Sultan ${ }^{1}$, Ok Joo Lee ${ }^{1}$, Ji Seung Lee ${ }^{1}$, Sung-il Yoon ${ }^{3}$, In-Sun Hong ${ }^{4}$, Gilson Khang ${ }^{5}$, Sang Jin Lee (1) ${ }^{6}$, James J. Yoo ${ }^{6} \&$ Chan Hum Park ${ }^{1,7}$

Correction to: Nature Communications https://doi.org/10.1038/s41467-018-03759-y, published online 24 April 2018

The original version of this Article contained errors in Figs. 5 and 6. In Fig. 5b, the second panel on the bottom row was stretched out of proportion. The correct version of Fig. $5 \mathrm{~b}$ appears below as Fig. 1:

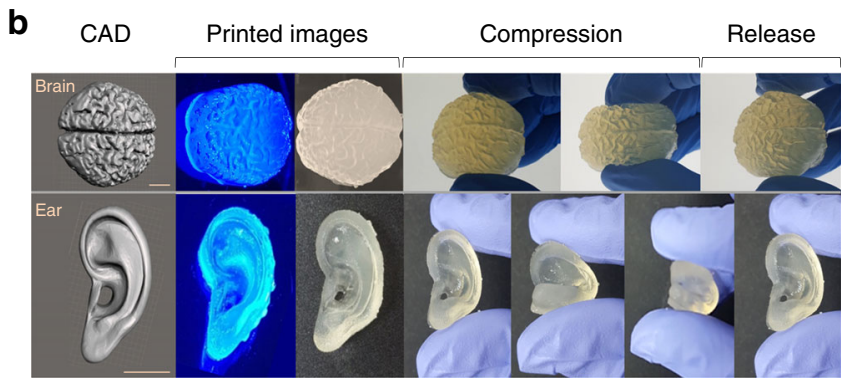

Fig. 1 .

which replaces the previous incorrect version that appears as Fig. 2 below.

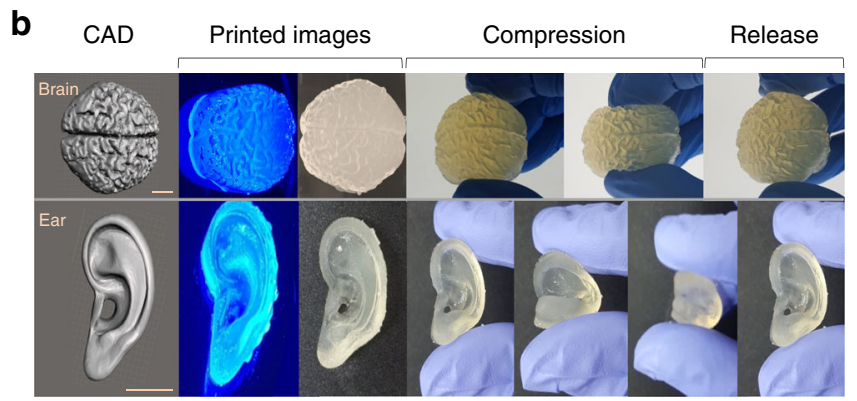

Fig. 2

\footnotetext{
${ }^{1}$ Nano-Bio Regenerative Medical Institute, College of Medicine, Hallym University, Chuncheon 24252, Republic of Korea. ${ }^{2}$ School of Medicine, George Washington University, Washington, DC 20037, USA. ${ }^{3}$ Division of Biomedical Convergence, College of Biomedical Science, Kangwon National University, Chuncheon 24341, Republic of Korea. ${ }^{4}$ Department of Molecular Medicine, School of Medicine, Gachon University, Incheon 406-840, Republic of Korea. ${ }^{5}$ Department of BIN Convergence Technology, Department of Polymer Nano Science \& Technology and Polymer Materials Fusion Research Center, Chonbuk National University, Jeonju 54896, Republic of Korea. ${ }^{6}$ Wake Forest Institute for Regenerative Medicine, Wake Forest School of Medicine, Medical Center Boulevard, Winston-Salem, NC 27157, USA. ${ }^{7}$ Departments of Otorhinolaryngology-Head and Neck Surgery, Chuncheon Sacred Heart Hospital, School of Medicine, Hallym University, Chuncheon 24252, Republic of Korea. These authors contributed equally: Soon Hee Kim, Yeung Kyu Yeon, Jung Min Lee. Correspondence and requests for materials should be addressed to C.H.P. (email: hlpch@paran.com)
} 
In Fig. 6d, the first panel was also stretched out of proportion. In Fig. 6f, the fifth panel inadvertently repeated the fourth. The correct version of Fig. $6 \mathrm{c}-\mathrm{f}$ which appears below as Fig. 3:

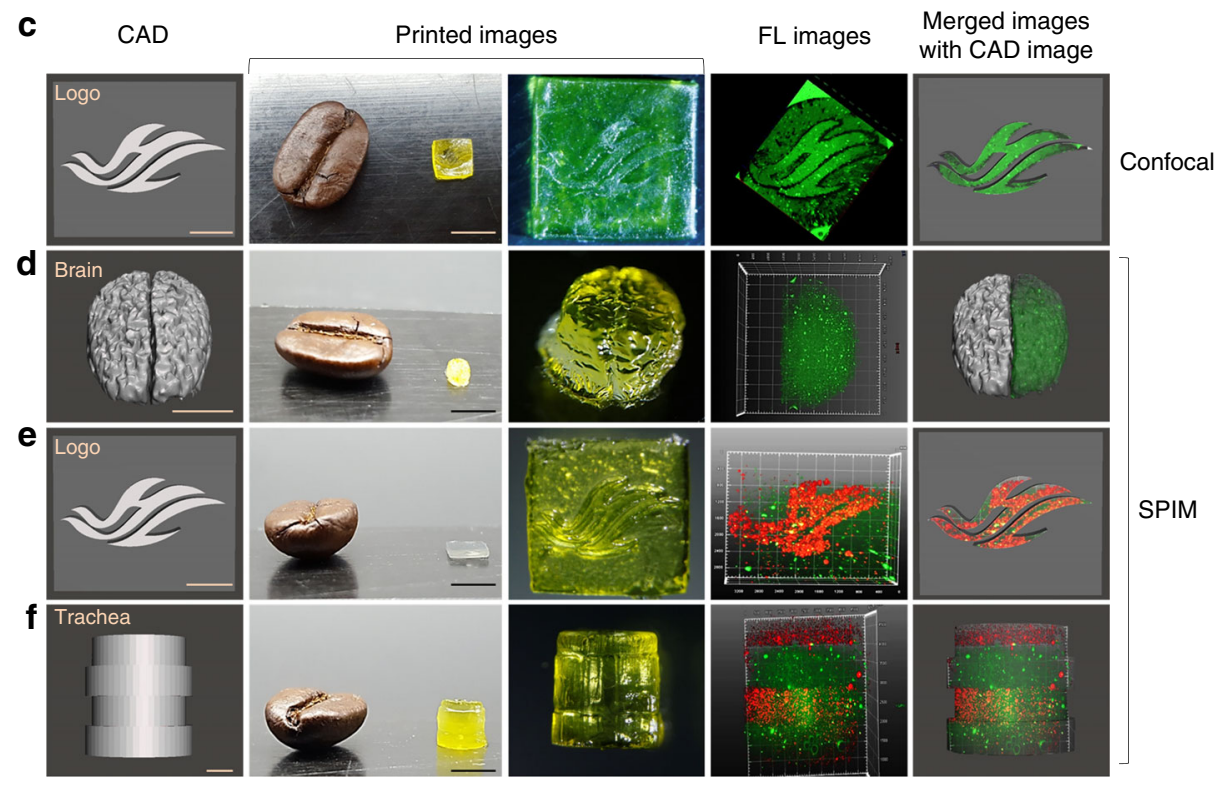

Fig. 3

which replaces the previous incorrect version that appears as Fig. 4 below.

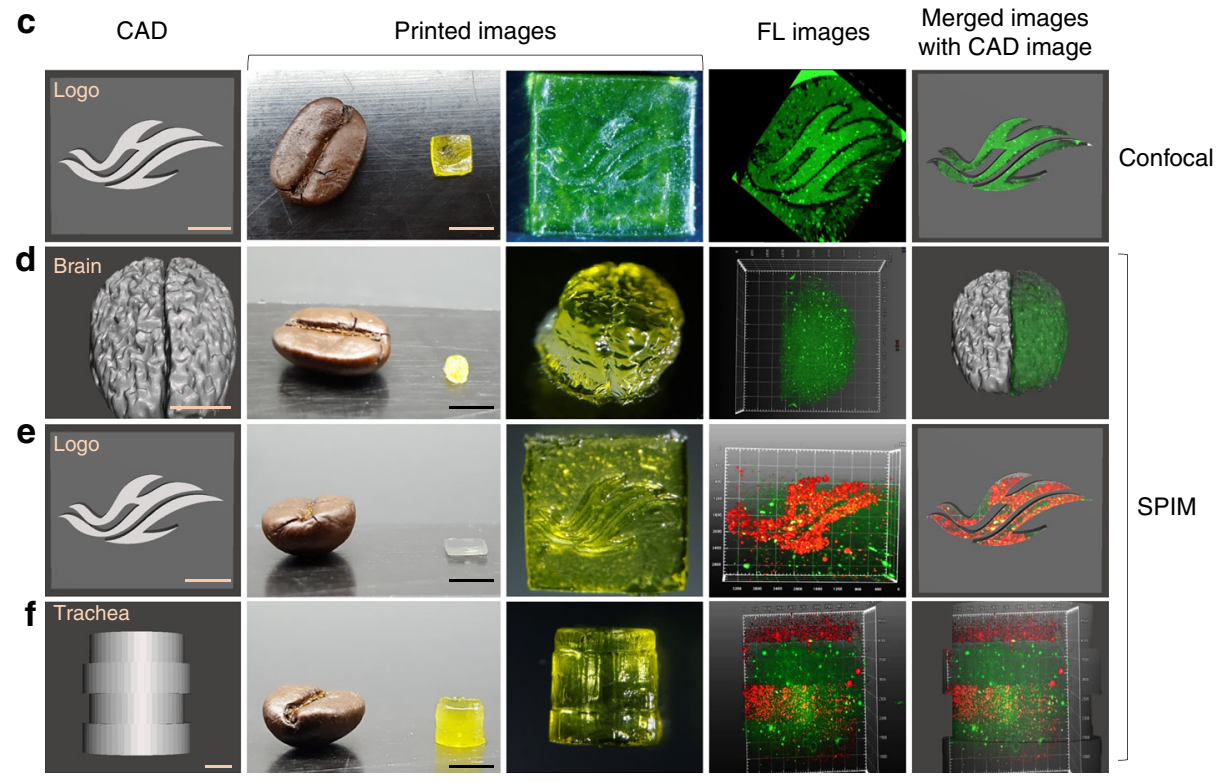

Fig. 4 .

This has been corrected in both the PDF and HTML versions of the Article.

Published online: 11 June 2018

(i) Open Access This article is licensed under a Creative Commons Attribution 4.0 International License, which permits use, sharing, adaptation, distribution and reproduction in any medium or format, as long as you give appropriate credit to the original author(s) and the source, provide a link to the Creative Commons license, and indicate if changes were made. The images or other third party material in this article are included in the article's Creative Commons license, unless indicated otherwise in a credit line to the material. If material is not included in the article's Creative Commons license and your intended use is not permitted by statutory regulation or exceeds the permitted use, you will need to obtain permission directly from the copyright holder. To view a copy of this license, visit http://creativecommons.org/licenses/by/4.0/. 